\title{
Article \\ Prognostic Factors for Mortality, Activity of Daily Living, and Quality of Life in Taiwanese Older Patients within 1 Year Following Hip Fracture Surgery
}

\author{
Ming-Hsiu Chiang ${ }^{1,+}(\mathbb{D})$, Yu-Yun Huang ${ }^{2,+}$, Yi-Jie Kuo ${ }^{3,4,+} \mathbb{D}$, Shu-Wei Huang ${ }^{3} \mathbb{D}$, Yeu-Chai Jang ${ }^{5}$, \\ Fu-Ling Chu ${ }^{2, *(D)}$ and Yu-Pin Chen ${ }^{3,4, *(\mathbb{D}}$
}

Citation: Chiang, M.-H.;

Huang, Y.-Y.; Kuo, Y.-J.; Huang, S.-W.;

Jang, Y.-C.; Chu, F.-L.; Chen, Y.-P.

Prognostic Factors for Mortality,

Activity of Daily Living, and Quality

of Life in Taiwanese Older Patients

within 1 Year Following Hip Fracture

Surgery. J. Pers. Med. 2022, 12, 102.

https://doi.org/10.3390/

jpm12010102

Academic Editors: Maximillian

Rudert and Pedro Berjano

Received: 21 November 2021

Accepted: 11 January 2022

Published: 13 January 2022

Publisher's Note: MDPI stays neutral with regard to jurisdictional claims in published maps and institutional affiliations.

Copyright: () 2022 by the authors. Licensee MDPI, Basel, Switzerland. This article is an open access article distributed under the terms and conditions of the Creative Commons Attribution (CC BY) license (https:// creativecommons.org/licenses/by/ $4.0 /)$.
1 Department of General Medicine, Kaohsiung Chang Gung Memorial Hospital, Kaohsiung 833, Taiwan; b101103050@tmu.edu.tw

2 Department of Nursing \& Graduate Institute of Nursing, Chang Gung University of Science and Technology, Kweishan, Taoyuan 333, Taiwan; cutefloat@hotmail.com

3 Department of Orthopedic Surgery, Wan Fang Hospital, Taipei Medical University, Taipei 116, Taiwan; benkuo5@gmail.com (Y.-J.K.); judyya1022@gmail.com (S.-W.H.)

4 Department of Orthopedic Surgery, School of Medicine, College of Medicine, Taipei Medical University, Taipei 110, Taiwan

5 Department of Obstetrics and Gynecology, Wan Fang Hospital, Taipei Medical University, Taipei 116, Taiwan; Chvicky1107@gmail.com

* Correspondence: fchu@mail.cgust.edu.tw (F.-L.C.); 99231@w.tmu.edu.tw (Y.-P.C.); Tel.: +886-03-2118999 (F.-L.C.); Fax: +886-03-2118866 (F.-L.C.)

+ These authors contributed equally to this work.

\begin{abstract}
Background. Hip fractures among older adults are a major public health concern worldwide This study investigated the potential clinical factors that predict postoperative 1-year activities of daily living (ADL), quality of life (QoL), and mortality in Taiwanese older adults following hip fracture. Methods. This is a prospective cohort study enrolling older adults ( $\geq 60$ years) who had undergone hip fracture surgery in a single medical center. The comprehensive clinical history of each patient was examined. QoL, ADL, and mortality events were recorded consecutively at 3, 6, and 12 months after operation. The multiple logistic regression model and the generalized estimating equation (GEE) were adopted to identify contributing factors for mortality and postoperative ADL and QoL prognosis, respectively. Results. Among 377 participants with hip fracture, 48 died within 1 year of the index operation. ADL and QoL considerably decreased at 3 months following hip surgery. Old age, high Charlson Comorbidity Index, and American Society of Anesthesiologists grading were crucial predictors for mortality at the 1-year follow-up. The generalized estimating equation analysis indicated that the length of postoperative follow-up time, serum albumin level, patient cognitive status, and handgrip strength were considerably associated with QoL and ADL recovery prognosis in the Taiwanese older adults following hip fracture. Conclusions. Hip fractures have long-lasting effects on the older adults. Our data imply several prognosis predicting parameters that may assist clinicians in accounting for an individual's personalized risks in order to improve functional outcomes and reduce mortality.
\end{abstract}

Keywords: prognostic factors; hip fracture; mortality; activity of daily living; quality of life; older adult assessment

\section{Introduction}

Hip fracture is a debilitating disease among older adults [1] and its incidence is increasing. In Taiwan, the incidence of all types of hip fractures increased by $8.6 \%$ between 2000 and 2010. In 2018, Taiwan officially became an aged society, and the estimated total number of hip fractures in Taiwan was projected to increase from 18,338 in 2010 to 50,421 by 2035 [2]. Direct costs of hip fracture treatment are enormous, and its 
subsequent negative effects, including disability, higher risk of cardiovascular disease, and depression, impose a great burden on the patient's family and are of considerable public health concern [3].

The prognosis of older adults after hip fractures is poor. Activities of daily living (ADLs) and quality of life (QoL) deteriorate significantly after 6 months [4], and the mortality rate is approximately $5.5 \%$ at 3 months and $9.1 \%$ at 6 months [4] and can be as high as $36 \%$ at 1 year postoperatively [5]. Coronary heart disease, pneumonia, and urinary tract infection are major risk factors for mortality in older adults with hip fracture [6]. In terms of functional recovery, only approximately one-fifth of older adults with hip fractures recover their preinjury functional status 1 year postoperatively $[4,7]$. Therefore, it is critical to identify hip fracture risk factors and organize public health programs to prevent hip fractures.

In patients with hip fracture, postoperative functional recovery and QoL are highly associated with multiple variables, including age, sex, medical complications, baseline Charlson Comorbidity Index (CCI), and sarcopenia [8-10]. The influence of surgical delay on postoperative prognosis was deleterious. Mortality significantly increased for patients receiving hip repair surgery $>24$ hours after admission compared with those operated on within 24 hours [11]. A surgical delay of $>21$ days had a significantly poorer hip function and QoL than an operation within 7 days of the fracture [12]. Moreover, delays in surgery are correlated with a longer length of hospitalization and a lower possibility of returning to independent living [13]. Handgrip strength is a critical indicator of muscle strength and short-term functional outcome prediction [14]. Predictors of postoperative mortality in patients with hip fracture include age, sex, underlying comorbidities, high American Society of Anesthesiologists (ASA) grading, and prefracture mobility [15]. Patients with poor renal function at presentation are also at an increased risk of death and other complications, including renal, pulmonary, and thromboembolic diseases [16].

With a knowledge of prognostic factors, clinicians can adopt a stratified care approach by prioritizing older adults with hip fractures at a high risk of poor outcomes or high mortality for intensive care [17]. However, considerable variations have been observed among studies in prognostic factors for outcomes following hip fracture surgery in older adults. In addition, most studies have only calculated the changes in ADL or QoL between the preoperative level and that at the last follow-up and may thus have neglected other potential predicting variables if the analysis was conducted in a consecutive manner. This prospective study recorded preinjury and postoperative ADL and QoL at 3, 6, and 12 months and identified the independent prognostic factors of mortality and changes in ADL and QoL in patients with hip fractures aged $\geq 60$ years.

\section{Methods}

\subsection{Study Design}

This prospective cohort study recruited older adults who underwent surgery for hip fracture at a single medical center in Taiwan from 1 January 2017 to 31 December 2019. Qualifying patients were men and women aged $\geq 60$ years who had hip fracture, including intracapsular femoral neck fracture and extracapsular (i.e., basal neck, intertrochanteric, or subtrochanteric) fracture. Patients were excluded if they underwent hip surgery because of a condition other than primary hip fracture, such as osteoarthritis, trauma, tumor metastasis, infection, or avascular necrosis of the femoral head. The study complied with the code of ethics of the World Medical Association (Declaration of Helsinki) and was approved by the Ethics Committee of Taipei Medical University (TMU-JIRB N201709053). Informed consent was obtained from all the participants for publication and participation in the study. 


\subsection{Measurement of Handgrip Strength}

Isometric grip strength, which is the maximum handgrip strength, was measured using a Jamar hydraulic dynamometer (Sammons Preston, Bolingbrook, IL, USA) while the patient was sitting in the bed or on a chair, with the elbow flexed and the wrist in the neutral position, with verbal encouragement. Patients were instructed to squeeze the device three times in each hand as hard as possible [18]. The same investigator conducted measurements for all participants and was blind to the clinical data. For each participant, the best of six measurements was used. A handgrip strength of $<26 \mathrm{~kg}$ for men and $<18 \mathrm{~kg}$ for women is considered low, based on the threshold values recommended by the Asian Working Group for Sarcopenia [19].

\subsection{Measurement of Other Clinical Parameters}

Basic demographic data, including age, sex, and body mass index, were collected from medical records for analysis, along with preoperative laboratory data, including hemoglobin level, serum creatinine, sodium, and albumin levels. The underlying comorbidities were presented as CCI. We also recorded the specific amount of time elapsed between a patient's fall and the time of operation. The operation records of each patient were screened to extract the following information: ASA grade, type of hip fracture repair surgery, and blood loss during the operation. Bone mineral density (BMD) T-score and handgrip strength were obtained within 1 week after surgery (i.e., T-score obtained through dual-energy X-ray absorptiometry (DXA)) [10]. After patient consent was obtained, interviews were conducted with the patients and their caregivers on the patients' admission for hip fracture surgery by using the Short Portable Mental Status Questionnaire (SPMSQ) [20] for screening dementia. For assessing preinjury and postoperative (at 3, 6, and 12 months) performance in ADL and QoL, the Barthel Index (BI) and EuroQol-5D questionnaire (EQ-5D) [21] were adopted, respectively [22]. The BI has an ordinal scale with scores from 0 to 100 and is calculated using 10 variables that represent ADL and mobility [22]. A higher value is associated with a greater likelihood of independent community living. The Chinese version of the EQ5D was used in this study and it exhibited a high level of agreement (intraclass correlation coefficients $>0.75$ ) and convergent validity (Pearson's correlation coefficients $>0.95$ ) with the value sets of the versions of the EQ-5D from the UK, Japan, and Korea [23]. The Chinese version of the BI has been validated with moderate to excellent agreement among raters for individual items (kappa: 0.53-0.94) and total score (intraclass correlation coefficient Z: 0.94) [24].

\section{Statistical Analysis}

All statistical analyses were conducted using SPSS Statistics for Windows, version 18.0 (SPSS, Chicago, IL, USA). Categorical variables are presented as frequencies and percentages and continuous variables are presented as mean \pm standard deviation. Univariate analyses were conducted on numerous potential risk factors for mortality between survival and mortality subgroups 1 year after hip fracture surgery; a chi-squared test was used to compare categorical variables, whereas an independent t-test was used to compare continuous variables. Factors that differed significantly $(p<0.05)$ in the univariate analysis were included in the multiple logistic regression model to identify critical mortality-contributing factors. In terms of comparing repeated ADL measurements at postoperative 3, 6, and 12 months with preinjury status, a paired $t$-test was used. Postoperative EQ-5D and BI were measured repetitively, so a generalized estimating equation (GEE) was adopted to screen all potential influencing clinical parameters. For cases that were lost to follow-up, missing data imputation was performed. For all tests, two-sided $p<0.05$ was considered statistically significant.

\section{Results}

A total of 416 older adults were screened; two were suspected of pathologic fracture, four of femoral head avascular necrosis, 15 were less than 60 years old, and 18 refused to 
participate. At last, 377 older adults with hip fracture, comprising 105 men (28\%) and 272 women $(72 \%)$, were enrolled. Supplementary Figure S1 shows the study flowchart and number of patients staying in contact at each follow-up. Overall, 56 participants lost contact after 1 year of follow-up, resulting in a 1-year loss to follow-up rate of around $14.8 \%$. The mean age was $81 \pm 9.5$ years (range: $60-103$ years). The average body mass index was $22 \pm 3.6$, and the mean CCI was $4.8 \pm 1.8$. Of the 377 patients, 203 had femoral neck fracture (54\%) and 174 had intertrochanteric fracture (46\%). Upon admission, the patients had a mean handgrip strength of $12 \pm 9.4 \mathrm{~kg}$. The average ASA grade was $2.7 \pm 0.5$, and the mean surgical delay from the index fracture was $77 \pm 234 \mathrm{~h}$. Furthermore, 236 patients (63\%) received open reduction and internal fixation, and the remaining underwent joint replacement surgery. Table 1 summarizes the patients baseline clinicodemographic characteristics.

Table 1. Baseline clinicodemographic characteristics of the study population.

\begin{tabular}{|c|c|}
\hline Clinical Characteristics $(n=377)$ & Mean \pm SD/Number (Percentage) \\
\hline Age & $81 \pm 9.5$ \\
\hline \multicolumn{2}{|l|}{ Gender } \\
\hline Male & $105(28 \%)$ \\
\hline Female & $272(72 \%)$ \\
\hline BMI & $22 \pm 3.6$ \\
\hline CCI & $4.8 \pm 1.8$ \\
\hline \multicolumn{2}{|l|}{ Types of fracture } \\
\hline Femoral neck fracture & $203(54 \%)$ \\
\hline Pertrochanteric fracture & $174(46 \%)$ \\
\hline ASA grading & $2.7 \pm 0.52$ \\
\hline SPMSQ score & $3.5 \pm 3.7$ \\
\hline Handgrip strength (kg) & $12 \pm 9.4$ \\
\hline BMD T-score & $-3.8 \pm 1.1$ \\
\hline \multicolumn{2}{|l|}{ Pre-operation laboratory data } \\
\hline $\mathrm{Hb}(\mathrm{g} / \mathrm{dL})$ & $12 \pm 2.3$ \\
\hline $\mathrm{Na}(\mathrm{mmol} / \mathrm{L})$ & $137 \pm 4.1$ \\
\hline Creatinine $(\mathrm{mg} / \mathrm{dL})$ & $1.2 \pm 1.2$ \\
\hline \multicolumn{2}{|l|}{$\begin{array}{l}\text { vitamin D3 (ng/mL) } \\
(n=260)\end{array}$} \\
\hline$(n=308)$ & $55 \pm 62$ \\
\hline$(n=336)$ & $3.1 \pm 0.4$ \\
\hline \multicolumn{2}{|l|}{ Surgical record } \\
\hline Surgical delay (hour) & $77 \pm 234$ \\
\hline \multicolumn{2}{|l|}{265 patients $(70 \%)<48 \mathrm{~h}$} \\
\hline \multicolumn{2}{|l|}{294 patients $(78 \%) \leq 72 \mathrm{~h}$} \\
\hline Operation time (min) & $79 \pm 41$ \\
\hline Blood loss (cc) & $111 \pm 109$ \\
\hline \multicolumn{2}{|l|}{ Surgical classification } \\
\hline ORIF & $236(63 \%)$ \\
\hline Joint replacement & $141(37 \%)$ \\
\hline
\end{tabular}

Abbreviations: ASA, American Society of Anesthesiologists; BMI, body mass index; BMD, bone mineral density $\mathrm{CCI}$, Charlson Comorbidity Index; Hb, hemoglobin; ORIF, open reduction and internal fixation; SD, standard deviation; SPMSQ, Short Portable Mental Status Questionnaire.

In all, 48 patients died within 1 year after surgery; thus, the 1-year mortality rate after hip fracture was approximately $12.7 \%$. Univariate analysis revealed that the following clinical variables were significantly correlated with postoperative 1-year mortality: age, CCI, preoperative hemoglobin value, serum albumin level, SPMSQ score, handgrip strength, and ASA grade (Supplementary Table S1). However, only age, CCI, and ASA grade were significant in the multiple logistic regression model (Table 2). 
Table 2. Multiple logistic regression model of factors affecting postoperative 1-year mortality in older adults with hip fracture.

\begin{tabular}{cccccc}
\hline \multirow{2}{*}{ Variables } & \multirow{2}{*}{ B } & $p$-Value & Odds Ratio & \multicolumn{2}{c}{$\mathbf{9 5 \% \text { CI Odds Ratio }}$} \\
\cline { 5 - 6 } & & & & Lower & Upper \\
\hline Age & $\mathbf{0 . 0 7}$ & $\mathbf{0 . 0 1 6}$ & $\mathbf{1 . 0 7}$ & $\mathbf{1 . 0 1}$ & $\mathbf{1 . 1}$ \\
\hline CCI & $\mathbf{0 . 2 2}$ & $\mathbf{0 . 0 3}$ & $\mathbf{1 . 3}$ & $\mathbf{1 . 0 2}$ & $\mathbf{1 . 5}$ \\
\hline Pre-operation $\mathrm{Hb}(\mathrm{g} / \mathrm{dL})$ & -0.16 & 0.11 & 0.85 & 0.70 & 1.04 \\
\hline Albumin $(\mathrm{g} / \mathrm{dL})$ & -0.22 & 0.7 & 0.81 & 0.30 & 2.2 \\
\hline SPMSQ score & 0.09 & 0.09 & 1.1 & 0.97 & 1.2 \\
\hline Handgrip strength $(\mathrm{kg})$ & -0.04 & 0.3 & 0.96 & 0.89 & 1.04 \\
\hline ASA grading & $\mathbf{1 . 5}$ & $\mathbf{0 . 0 3}$ & $\mathbf{4 . 6}$ & $\mathbf{1 . 1}$ & $\mathbf{1 8 . 7}$
\end{tabular}

Abbreviations: ASA, American Society of Anesthesiologists; CCI, Charlson Comorbidity Index; CI, confidence interval; $\mathrm{Hb}$, hemoglobin; SPMSQ, Short Portable Mental Status Questionnaire.

A significant decrease in QoL and ADL was observed at postoperative 3, 6, and 12 months compared with the patients' preinjury status. The preinjury EQ-5D score was 0.84 and decreased to $0.73,0.76$, and 0.77 at postoperative 3,6 , and 12 months, respectively (all $p<0.001$; Figure 1), and BI decreased from 84.4 to $68.97,71.27$, and 71.11, respectively, without signs of recovery to their preinjury status (all $p<0.001$; Figure 2). We assessed the impact of different clinical parameters on postoperative EQ-5D scores and BI by using GEE. In the univariate analysis, age, CCI, BMD T-score, preoperative hemoglobin value, serum albumin level, SPMSQ score, ASA grade, length of postoperative follow-up time, and handgrip strength were independently associated with postoperative EQ-5D score and BI (Supplementary Tables S2 and S3). However, multivariate analysis with multiple imputations revealed that only the serum albumin level, SPMSQ score, handgrip strength, and length of postoperative follow-up time had a significant impact on both postoperative EQ-5D scores and BI (Table 3). Higher serum albumin level and handgrip strength were associated with better improvements in QoL and ADL, whereas higher SPMSQ score was inversely related to poorer outcomes. In terms of postoperative follow-up time, a significant reduction in QoL and ADL persisted up to postoperative 1 year compared with the patients' preinjury status.

\section{Changes on QoL: $\quad{ }^{*}: p<0.001$}

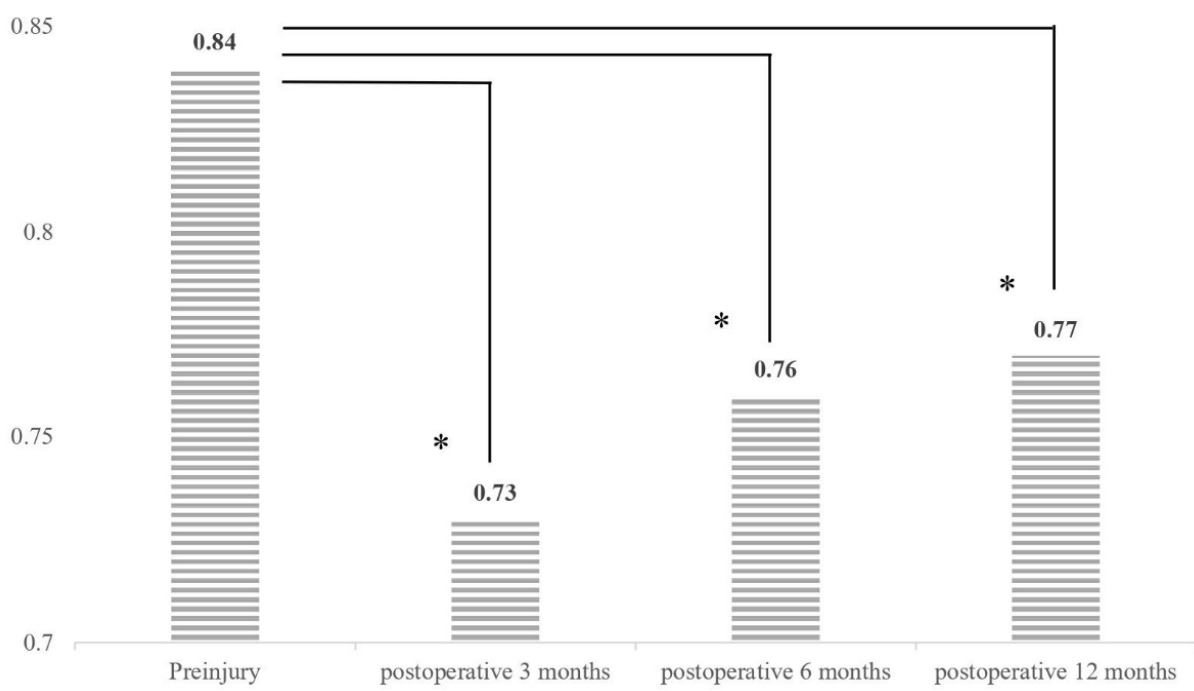

Figure 1. Consecutive QoL (EQ-5D) measurements at preinjury, 3, 6, and 12 months after hip fracture repair surgery. ${ }^{*}: p<0.001$. 


\section{Changes on ADL:}

90

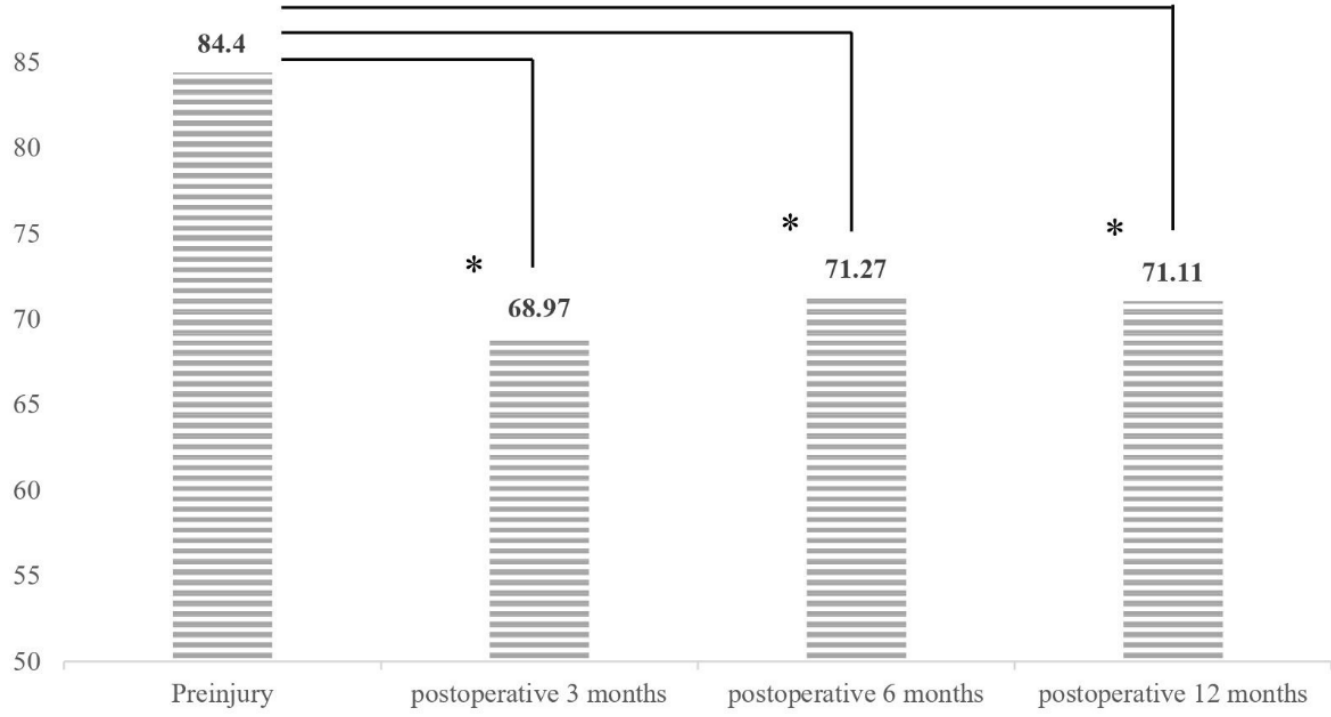

Figure 2. Consecutive ADL (BI) measurements at preinjury, 3, 6, and 12 months after hip fracture repair surgery. ${ }^{*}: p<0.001$.

Table 3. GEE model with multiple imputation of factors affecting postoperative EQ-5D and ADL in older adults with hip fracture.

\begin{tabular}{|c|c|c|c|c|}
\hline \multirow{2}{*}{ Variables } & \multirow{2}{*}{$\beta$} & \multirow{2}{*}{$p$-Value } & \multicolumn{2}{|c|}{ 95\% CI Ratio } \\
\hline & & & Lower & Upper \\
\hline \multicolumn{5}{|c|}{ EQ-5D } \\
\hline Postoperative 3 months vs. preinjury status & -0.31 & $<0.001$ & -0.35 & -0.27 \\
\hline Postoperative 6 months vs. preinjury status & -0.25 & $<0.001$ & -0.29 & -0.20 \\
\hline Postoperative 12 months vs. preinjury status & -0.23 & $<0.001$ & -0.27 & -0.18 \\
\hline Age & -0.001 & 0.34 & -0.004 & 0.001 \\
\hline CCI & -0.008 & 0.25 & -0.021 & 0.005 \\
\hline BMD T-score & 0.007 & 0.48 & -0.012 & 0.025 \\
\hline Pre-operation $\mathrm{Hb}(\mathrm{g} / \mathrm{dL})$ & 0.005 & 0.40 & -0.006 & 0.016 \\
\hline $\operatorname{Albumin}(\mathrm{g} / \mathrm{dL})$ & 0.060 & 0.018 & 0.010 & 0.11 \\
\hline SPMSQ & -0.019 & $<0.001$ & -0.025 & -0.013 \\
\hline ASA grading & 0.005 & 0.81 & -0.038 & 0.048 \\
\hline Handgrip strength $(\mathrm{kg})$ & 0.003 & 0.01 & 0.001 & 0.005 \\
\hline \multicolumn{5}{|c|}{ ADL } \\
\hline Postoperative 3 months vs. preinjury status & -32.6 & $<0.001$ & -36.8 & -28.4 \\
\hline Postoperative 6 months vs. preinjury status & -25.6 & $<0.001$ & -30.3 & -20.9 \\
\hline Postoperative 12 months vs. preinjury status & -25.5 & $<0.001$ & -30.2 & -20.8 \\
\hline Age & 0.11 & 0.43 & -0.16 & 0.39 \\
\hline CCI & -0.85 & 0.24 & -2.3 & 0.56 \\
\hline
\end{tabular}


Table 3. Cont.

\begin{tabular}{|c|c|c|c|c|}
\hline \multirow{2}{*}{ Variables } & \multirow{2}{*}{$\beta$} & \multirow{2}{*}{$p$-Value } & \multicolumn{2}{|c|}{ 95\% CI Ratio } \\
\hline & & & Lower & Upper \\
\hline BMD T-score & 0.66 & 0.52 & -1.34 & 2.7 \\
\hline Pre-operation $\mathrm{Hb}$ (g/dL) & 0.5 & 0.43 & -0.74 & 1.7 \\
\hline Albumin (g/dL) & 8.8 & 0.002 & 3.2 & 14.3 \\
\hline SPMSQ & -2.9 & $<0.001$ & -3.5 & -2.26 \\
\hline ASA grading & -2.1 & 0.38 & -6.9 & 2.6 \\
\hline Handgrip strength (kg) & 0.29 & 0.017 & 0.05 & 0.52 \\
\hline Hip fracture type (PTF vs. FNF) & -2.5 & 0.27 & -7.0 & 1.9 \\
\hline
\end{tabular}

Abbreviations: ASA, American Society of Anesthesiologists; BMD, bone mineral density; CCI, Charlson Comorbidity Index; CI, confidence interval; FNF, femoral neck fracture; Hb, hemoglobin; PTF, pertrochanteric fracture; SPMSQ, Short Portable Mental Status Questionnaire.

\section{Discussion}

Several key epidemiological observations were noted in the present study. The overall participant's mean age was 81 years old, with female predominance. Similarly, one epidemiological study from Taiwan indicated that the average age of hip fracture was $76.7 \pm 9.0$ years for women and $74.1 \pm 9.6$ years for men [25]. Anemia, malnutrition, and osteoporosis were prevalent in all participants. Moreover, the average SPMSQ score was 3.5, which indicated that over half of the total number of participants had mild dementia. Finally, the mean handgrip strength was $9.4 \mathrm{~kg}$ in the present study, which was much lower than the sarcopenia diagnostic criteria ( $<26 \mathrm{~kg}$ in men and $<18 \mathrm{~kg}$ in women), implying a high incidence of concomitant sarcopenia and hip fracture. Consistently, a recent crosssectional study reported that over half of older adults with hip fracture had sarcopenia [10]. In other words, older adults with hip fracture are more fragile and may thus require more healthcare resources.

Apart from old age [26], various clinical variables have been regarded as risk factors for mortality after hip fracture repair surgery. Li et al. concluded that the odds of postoperative 1-year mortality was 3.35 in patients whose CCI scores were $>3$ [27]. In addition, patients with a CCI score of $\geq 3$ sustained an increased $19 \%$ risk of recurrent osteoporotic fracture compared with those with a CCI score of 0 at 1 year after the hip fracture [28]. Lower handgrip strength has also been associated with higher postoperative mortality [29]. However, in our study, handgrip strength was a significant predictor for postoperative mortality only in the univariate analysis and not in the multiple logistic regression model. This may be because the patients' overall handgrip strength was generally low. Furthermore, the high correlation between age, CCI, and ASA grade may be another possible reason $(\mathrm{R}=-0.28,-0.27$, and -0.24 , respectively; all $p<0.01)$ that overshadowed the predictability of handgrip strength. More well-organized prospective studies are warranted to determine the reliability of handgrip strength in predicting mortality after hip fracture repair surgery.

Hip fracture can considerably impair the QoL and mobility of older adults. Hershkovitz et al. reported that $<10 \%$ of patients with hip fracture are functionally independent after 1 year of postoperative rehabilitation [29]. Health-related QoL also severely deteriorates after hip fracture. Using the EQ-5D, Amarilla-Donoso et al. reported a significant reduction in all dimensions from prefracture status to postoperative 1 month [30]. In the present study, after 1 year of follow-up, we observed that both EQ-5D scores and BI reached the lowest level at postoperative 3 months. Although QoL and ADL later improved in most participants, they remained significantly lower than their preinjury status. 
Multiple clinical parameters have been proposed as predictors for postoperative ADL recovery, including age, sex, surgical delay, preinjury status, [31-33] preoperative hemoglobin values, renal function, and handgrip strength [34,35]. In the present study, nine clinical variables were identified to be independently associated with postoperative $\mathrm{BI}$ in the GEE univariate analysis. However, only the length of postoperative follow-up time, serum albumin level, SPMSQ score, and handgrip strength were found to be significant in the GEE multivariate analysis model with multiple imputation. Dementia, delirium, and malnutrition are also critical risk factors for poor postoperative outcomes following hip fracture [36,37]. Handgrip strength is assessed noninvasively and is thus convenient for repetitive measurements. It is not only a part of sarcopenia diagnostic criteria but has also been drawing attention as a prognostic indicator for postoperative ADL in recent years. Choi et al. enrolled 242 older adults with hip fracture and recorded their respective handgrip strength with a hand dynamometer. They reported that handgrip strength is predictive of postoperative complications and can therefore be useful for preoperative screening [38]. Di Monaco et al. also reported that handgrip strength assessed before rehabilitation can independently predict functional outcomes after a 6-month follow-up in women with hip fracture [39]. In summary, the role of handgrip strength as a prognostic factor after hip fracture in older adults is gaining attention. Our findings corroborate that handgrip strength is significantly correlated with postoperative ADL changes.

The role of sex in predicting postoperative ambulatory prognosis was heterogeneous. Some studies concluded sex is a major contributing factor [8,32]. Findings in the present study and others reported that man and woman had similar functional recovery or ability to return back to community lives [40,41]. We surmised that this discrepancy may be resulting from distinct population characteristics included in each study. For example, the mean age and number of comorbidities differed significantly between male and female, which could impact the potential for recovery. In the present study there were no significant differences in age or CCI between male and female population. Long surgical delay after a falling accident was an important predictor for mortality and morbidity risk. However, in the present study, delay in surgery from fracture was not found to be correlated with mortality or ADL and QoL prognosis. One possible underlying etiology was that nearly $80 \%$ of the total participants were quickly sent to our hospital after injury and they received operation within 72 hours following hip fracture; only a minority of patients had prolonged surgical delay. BMD T-score was only identified as significant clinical parameters in the univariate analysis. One possible reason was the instant osteoporosis treatment prescription once osteoporosis was diagnosed. A recent study has reported that osteoporosis therapy incurred significant benefits on the patient's functional recovery [41]. As a result, the heterogeneous impact of lower BMD T-score may be hindered by the prompt initiation of osteoporosis treatment.

\section{Limitations}

This study has several limitations. First, only 377 participants were enrolled, and they may not represent all the older adults with hip fracture in Taiwan. Second, loss to follow-up is inevitable and when loss to follow-up exceeds $20 \%$, it may pose serious threats to the study validity [42]. In the present study, 1-year loss to follow-up rate was around $14.8 \%$ and missing data imputation was performed in GEE for compensation. However, the results should still be interpreted cautiously. Third, this study was conducted in a metropolitan area, and the findings may be region-specific and may not represent the general epidemiology of hip fracture in Taiwan. Finally, data were only collected before hip fracture surgery and within 1 year after surgery. Long-term follow-up studies are warranted to follow the natural course of hip fractures and their impact on QoL and motility in this specific cohort. 


\section{Conclusions and Implications}

By prospectively recruiting older adults who underwent surgery for hip fracture and following the postoperative changes in ADL and QoL, this study identified potential predicting clinical parameters. The postoperative 1-year mortality rate was approximately $12.7 \%$ in this study; both $\mathrm{BI}$ and EQ-5D declined severely at postoperative 3 months and recovered gradually afterwards. Age, CCI, and ASA grade were good predictors of postoperative mortality within 1 year. In addition, the length of postoperative followup time, serum albumin level, SPMSQ score, and handgrip strength were independent factors correlated with postoperative QoL and ADL; older adults continuously had poorer functional outcomes compared with their preinjury status up to 1 year after receiving hip fracture operation. Higher serum albumin level, hand grip strength and lower SPMSQ score were correlated with better postoperative QoL and ADL. Our findings may guide the establishment of a robust prediction and screening program that could improve functional outcomes and reduce the risk of mortality in older adults with hip fracture.

Supplementary Materials: The following supporting information can be downloaded at: https: / / www.mdpi.com/article/10.3390/jpm12010102/s1, Figure S1: Study profile; Table S1: Univariate analysis of potential clinical risk factors for postoperative 1-year mortality in older adults with hip fracture; Table S2: Univariate analysis of the GEE model with multiple imputation: potential risk factors affecting postoperative EQ-5D in older adults with hip fracture; Table S3: Univariate analysis of the GEE model with multiple imputation: factors affecting postoperative BI in older adults with hip fracture.

Author Contributions: Study concept and design: F.-L.C., Y.-J.K. and Y.-P.C.; acquisition of data: Y.-Y.H., Y.-C.J. and Y.-P.C.; analysis and interpretation of data: M.-H.C., Y.-Y.H. and S.-W.H.; drafting of the manuscript: M.-H.C., Y.-Y.H. and S.-W.H.; critical revision of the manuscript for important intellectual content: F.-L.C., Y.-J.K. and Y.-P.C. All authors have read and agreed to the published version of the manuscript.

Funding: The authors are grateful to Wan Fang Hospital (Grant numbers 111-wf-swf-07) and Medical University (Grant numbers TMU110-AE1-B07) for financially supporting this research.

Institutional Review Board Statement: The study complied with the code of ethics of the World Medical Association (Declaration of Helsinki) and was approved by the Ethics Committee of Taipei Medical University (TMU-JIRB N201709053).

Informed Consent Statement: Informed consent was obtained from all subjects involved in the study.

Data Availability Statement: Due to the sensitive nature of the questions asked in this study, survey respondents were assured that raw data would remain confidential and would not be shared.

Acknowledgments: The authors would like to acknowledge the Laboratory Animal Center at Taipei Medical University (TMU) for English editing service.

Conflicts of Interest: The authors declare that there is no conflict of interest.

\section{References}

1. Chiang, M.H.; Kuo, Y.J.; Chen, Y.P. The Association between Sarcopenia and Postoperative Outcomes among Older Adults with Hip Fracture: A Systematic Review. J. Appl. Gerontol. 2021, 40, 1903-1913. [CrossRef]

2. Chen, I.J.; Chiang, C.Y.; Li, Y.H.; Chang, C.H.; Hu, C.C.; Chen, D.W.; Chang, Y.; Yang, W.E.; Shih, H.N.; Ueng, S.W.; et al. Nationwide cohort study of hip fractures: Time trends in the incidence rates and projections up to 2035. Osteoporos. Int. 2015, 26, 681-688. [CrossRef] [PubMed]

3. Veronese, N.; Maggi, S. Epidemiology and social costs of hip fracture. Injury 2018, 49, 1458-1460. [CrossRef] [PubMed]

4. Chang, W.-T.; Kuo, Y.-J.; Huang, Y.-Y.; Tsai, M.-J.; Chen, Y.-P. Poor Activities of Daily Living Function Reflect Poor Quality of Life after Hip Fracture Surgery for Geriatric Patients. Soc. Health Behav. 2019, 2, 41-46.

5. Abrahamsen, B.; van Staa, T.; Ariely, R.; Olson, M.; Cooper, C. Excess mortality following hip fracture: A systematic epidemiological review. Osteoporos. Int. 2009, 20, 1633-1650. [CrossRef]

6. Koh, G.C.; Tai, B.C.; Ang, L.W.; Heng, D.; Yuan, J.M.; Koh, W.P. All-cause and cause-specific mortality after hip fracture among Chinese women and men: The Singapore Chinese Health Study. Osteoporos. Int. 2013, 24, 1981-1989. [CrossRef] 
7. Cornwall, R.; Gilbert, M.S.; Koval, K.J.; Strauss, E.; Siu, A.L. Functional outcomes and mortality vary among different types of hip fractures: A function of patient characteristics. Clin. Orthop. Relat. Res. 2004, 425, 64-71. [CrossRef]

8. Arinzon, Z.; Shabat, S.; Peisakh, A.; Gepstein, R.; Berner, Y.N. Gender differences influence the outcome of geriatric rehabilitation following hip fracture. Arch. Gerontol. Geriatr. 2010, 50, 86-91. [CrossRef] [PubMed]

9. Coto Carames, L.; Codesido Vilar, P.I.; Bravo Perez, M.; Mendoza Revilla, G.A.; Ojeda-Thies, C.; Blanco Hortas, A.; Quevedo Garcia, L.A. Influence of surgical parameters on mortality after surgery for extracapsular hip fractures in the elderly. Rev. Esp. Cir. Ortop. Traumatol. 2020, 64, 342-349. [CrossRef]

10. Chen, Y.-P.; Wong, P.-K.; Tsai, M., Jr.; Chang, W.-C.; Hsieh, T.-S.; Leu, T.-H.; Jeff Lin, C.-F.; Lee, C.-H.; Kuo, Y.-J.; Lin, C.-Y. The high prevalence of sarcopenia and its associated outcomes following hip surgery in Taiwanese geriatric patients with a hip fracture. $J$. Formos. Med. Assoc. 2020, 119, 1807-1816. [CrossRef]

11. Welford, P.; Jones, C.S.; Davies, G.; Kunutsor, S.K.; Costa, M.L.; Sayers, A.; Whitehouse, M.R. The association between surgical fixation of hip fractures within 24 hours and mortality: A systematic review and meta-analysis. Bone Jt. J. 2021, 103-b, 1176-1186. [CrossRef] [PubMed]

12. Song, J.; Zhang, G.; Liang, J.; Bai, C.; Dang, X.; Wang, K.; He, C.; Liu, R. Effects of delayed hip replacement on postoperative hip function and quality of life in elderly patients with femoral neck fracture. BMC Musculoskelet. Disord. 2020, 21, 487. [CrossRef]

13. Al-Ani, A.N.; Samuelsson, B.; Tidermark, J.; Norling, A.; Ekstrom, W.; Cederholm, T.; Hedstrom, M. Early operation on patients with a hip fracture improved the ability to return to independent living. A prospective study of 850 patients. J. Bone Jt. Surg. Am. Vol. 2008, 90, 1436-1442. [CrossRef] [PubMed]

14. Di Monaco, M.; Castiglioni, C.; De Toma, E.; Gardin, L.; Giordano, S.; Di Monaco, R.; Tappero, R. Handgrip strength but not appendicular lean mass is an independent predictor of functional outcome in hip-fracture women: A short-term prospective study. Arch. Phys. Med. Rehabil. 2014, 95, 1719-1724. [CrossRef] [PubMed]

15. Smith, T.; Pelpola, K.; Ball, M.; Ong, A.; Myint, P.K. Pre-operative indicators for mortality following hip fracture surgery: A systematic review and meta-analysis. Age Ageing 2014, 43, 464-471. [CrossRef]

16. Ramamurti, P.; Fassihi, S.C.; Sacolick, D.; Gu, A.; Wei, C.; Chodos, M.D. Impaired renal function is an independent risk factor for complications after surgery for femoral neck fracture. Hip Int. J. Clin. Exp. Res. Hip Pathol. Ther. 2021, epub ahead of print.

17. Penrod, J.D.; Litke, A.; Hawkes, W.G.; Magaziner, J.; Koval, K.J.; Doucette, J.T.; Silberzweig, S.B.; Siu, A.L. Heterogeneity in hip fracture patients: Age, functional status, and comorbidity. J. Am. Geriatr. Soc. 2007, 55, 407-413. [CrossRef]

18. Wang, C.Y.; Olson, S.L.; Protas, E.J. Test-retest strength reliability: Hand-held dynamometry in community-dwelling elderly fallers. Arch. Phys. Med. Rehabil. 2002, 83, 811-815. [CrossRef]

19. Chen, L.K.; Woo, J.; Assantachai, P.; Auyeung, T.W.; Chou, M.Y.; Iijima, K.; Jang, H.C.; Kang, L.; Kim, M.; Kim, S.; et al. Asian Working Group for Sarcopenia: 2019 Consensus Update on Sarcopenia Diagnosis and Treatment. J. Am. Med. Dir. Assoc. 2020, 21, 300-307.e2. [CrossRef]

20. Chi, I.; Boey, K.W. Hong Kong Validation of Measuring Instruments of Mental Health Status of the Elderly. Clin. Gerontol. 1993, 13, 35-51. [CrossRef]

21. EuroQol Group. EuroQol-a new facility for the measurement of health-related quality of life. Health Policy 1990, 16, 199-208. [CrossRef]

22. Mahoney, F.I.; Barthel, D.W. Functional evaluation: The barthel index. Md. State Med. J. 1965, 14, 61-65. [PubMed]

23. Wu, C.; Gong, Y.; Wu, J.; Zhang, S.; Yin, X.; Dong, X.; Li, W.; Cao, S.; Mkandawire, N.; Lu, Z. Chinese Version of the EQ-5D Preference Weights: Applicability in a Chinese General Population. PLoS ONE 2016, 11, e0164334. [CrossRef]

24. Hsueh, I.P.; Lee, M.M.; Hsieh, C.L. Psychometric characteristics of the Barthel activities of daily living index in stroke patients. J. Formos. Med. Assoc. 2001, 100, 526-532.

25. Chie, W.C.; Yang, R.S.; Liu, J.P.; Tsai, K.S. High incidence rate of hip fracture in Taiwan: Estimated from a nationwide health insurance database. Osteoporos. Int. 2004, 15, 998-1002. [CrossRef]

26. Aspray, T.J.; Hill, T.R. Osteoporosis and the Ageing Skeleton. Sub-Cell. Biochem. 2019, 91, 453-476.

27. Li, C.-M.; Li, C.-Y.; Chen, P.-C.; Chang, H.-H. Factors Associated with One-Year Mortality of Patients Receiving Home Healthcare Service in a Regional Hospital in Northern Taiwan. Taiwan J. Fam. Med. 2020, 30, 68-77.

28. Hsiao, P.-C.; Chen, T.-J.; Li, C.-Y.; Chu, C.-M.; Su, T.-P.; Wang, S.-H.; Pan, H.-H.; Wang, K.-Y. Risk factors and incidence of repeat osteoporotic fractures among the elderly in Taiwan: A population-based cohort study. Medicine 2015, 94, e532. [CrossRef] [PubMed]

29. Gutiérrez-Hermosillo, H.; de León-González, E.D.; Medina-Chávez, J.H.; Torres-Naranjo, F.; Martínez-Cordero, C.; Ferrari, S. Hand grip strength and early mortality after hip fracture. Arch. Osteoporos. 2020, 15, 185. [CrossRef] [PubMed]

30. Amarilla-Donoso, F.J.; Lopez-Espuela, F.; Roncero-Martin, R.; Leal-Hernandez, O.; Puerto-Parejo, L.M.; Aliaga-Vera, I.; ToribioFelipe, R.; Lavado-Garcia, J.M. Quality of life in elderly people after a hip fracture: A prospective study. Health Qual. Life Outcomes 2020, 18, 71. [CrossRef]

31. Sheehan, K.J.; Williamson, L.; Alexander, J.; Filliter, C.; Sobolev, B.; Guy, P.; Bearne, L.M.; Sackley, C. Prognostic factors of functional outcome after hip fracture surgery: A systematic review. Age Ageing 2018, 47, 661-670. [CrossRef]

32. Fukui, N.; Watanabe, Y.; Nakano, T.; Sawaguchi, T.; Matsushita, T. Predictors for ambulatory ability and the change in ADL after hip fracture in patients with different levels of mobility before injury: A 1-year prospective cohort study. J. Orthop. Trauma 2012, 26, 163-171. [CrossRef] 
33. Maggi, S.; Siviero, P.; Wetle, T.; Besdine, R.W.; Saugo, M.; Crepaldi, G.; Hip Fracture Study, G. A multicenter survey on profile of care for hip fracture: Predictors of mortality and disability. Osteoporos. Int. 2010, 21, 223-231. [CrossRef] [PubMed]

34. Ryan, G.; Nowak, L.; Melo, L.; Ward, S.; Atrey, A.; Schemitsch, E.H.; Nauth, A.; Khoshbin, A. Anemia at Presentation Predicts Acute Mortality and Need for Readmission Following Geriatric Hip Fracture. JB JS Open Access 2020, 5, e20.00048. [CrossRef]

35. Xu, B.Y.; Yan, S.; Low, L.L.; Vasanwala, F.F.; Low, S.G. Predictors of poor functional outcomes and mortality in patients with hip fracture: A systematic review. BMC Musculoskelet. Disord. 2019, 20, 568. [CrossRef]

36. Low, S.; Wee, E.; Dorevitch, M. Impact of place of residence, frailty and other factors on rehabilitation outcomes post hip fracture. Age Ageing 2021, 50, 423-430. [CrossRef] [PubMed]

37. O'Leary, L.; Jayatilaka, L.; Leader, R.; Fountain, J. Poor nutritional status correlates with mortality and worse postoperative outcomes in patients with femoral neck fractures. Bone Jt. J. 2021, 103-B, 164-169. [CrossRef]

38. Choi, J.Y.; Kim, J.K.; Kim, K.I.; Lee, Y.K.; Koo, K.H.; Kim, C.H. How does the multidimensional frailty score compare with grip strength for predicting outcomes after hip fracture surgery in older patients? A retrospective cohort study. BMC Geriatr. 2021, 21, 234. [CrossRef]

39. Di Monaco, M.; Castiglioni, C.; De Toma, E.; Gardin, L.; Giordano, S.; Tappero, R. Handgrip strength is an independent predictor of functional outcome in hip-fracture women: A prospective study with 6-month follow-up. Medicine 2015, 94, e542. [CrossRef] [PubMed]

40. Beaupre, L.A.; Carson, J.L.; Noveck, H.; Magaziner, J. Recovery of Walking Ability and Return to Community Living within 60 Days of Hip Fracture Does Not Differ Between Male and Female Survivors. J. Am. Geriatr. Soc. 2015, 63, 1640-1644. [CrossRef]

41. Orwig, D.L.; Abraham, D.S.; Hochberg, M.C.; Gruber-Baldini, A.; Guralnik, J.M.; Cappola, A.R.; Golden, J.; Hicks, G.E.; Miller, R.R.; Resnick, B.; et al. Sex Differences in Recovery across Multiple Domains among Older Adults with Hip Fracture. J. Gerontol. Ser. A 2021, glab271, epub ahead of print.

42. Dettori, J.R. Loss to follow-up. Evid. Based Spine Care J. 2011, 2, 7-10. [CrossRef] 